University of Nebraska - Lincoln

DigitalCommons@University of Nebraska - Lincoln

January 1973

\title{
Absolute line strengths by analysis of Lu-Fano plots with application to excited state transitions in neon
}

Anthony F. Starace

University of Nebraska-Lincoln, astarace1@unl.edu

Follow this and additional works at: https://digitalcommons.unl.edu/physicsstarace

Part of the Physics Commons

Starace, Anthony F., "Absolute line strengths by analysis of Lu-Fano plots with application to excited state transitions in neon" (1973). Anthony F. Starace Publications. 121.

https://digitalcommons.unl.edu/physicsstarace/121

This Article is brought to you for free and open access by the Research Papers in Physics and Astronomy at DigitalCommons@University of Nebraska - Lincoln. It has been accepted for inclusion in Anthony F. Starace Publications by an authorized administrator of DigitalCommons@University of Nebraska - Lincoln. 


\title{
Absolute line strengths by analysis of Lu-Fano plots with application to excited state transitions in neon
}

\author{
Anthony F. Starace \\ Department of Physics, The University of Chicago, Chicago, Illinois 60637 USA and Department of \\ Physics, Imperial College of Science and Technology, London SW7 \\ Current address: Behlen Laboratory of Physics, The University of Nebraska-Lincoln, Lincoln, Nebraska \\ 68508.
}

\begin{abstract}
Using energy eigenfunctions obtained by semi-empirical analysis of the Lu-Fano plot of energy level positions and employing the Coulomb approximation of Bates and Damgaard, we calculate absolute line strengths for transitions between the neon two-channel Rydberg series $2 \mathrm{p}^{5}\left({ }^{2} \mathrm{P}_{3 / 2,1 / 2}\right) n s J=1$ and $2 \mathrm{p}^{5}\left({ }^{2} \mathrm{P}_{3 / 2,1 / 2}\right) n^{\prime} \mathrm{p} J=0$ for $n, n^{\prime}=3$,4. Each Rydberg series is analyzed separately to obtain a set of five parameters which completely define the energy eigenfunctions for all values of $n$ and $n$ '. Interactions between the $n s J=1$ and the $n d J=1$ Rydberg series have been ignored. The one-channel neon $\mathrm{p}$ and s series are also discussed and line strengths for transition between them presented. Our line strengths for $3 s \rightarrow 3 p$ and $4 s \rightarrow 4 p$ transitions are in excellent agreement with the experimental and theoretical work of others. Our results for $3 \mathrm{~s} \rightarrow 4 \mathrm{p}$ and $4 \mathrm{~s} \rightarrow 3 \mathrm{p}$ transitions are not in agreement with previous work and differ substantially with intermediate coupling theory. For our application to low-lying energy levels in neon we have had to correct the formulas of Lu and Fano by inclusion of the energy-dependent factors of Seaton and by assuming a linear dependence on energy of intrinsic scattering phase shifts. We present all formulas necessary for computing absolute line strengths for transitions between multi-channel Rydberg series.
\end{abstract}

\section{Introduction}

The Coulomb approximation of Bates and Damgaard (1949) has been used successfully for the calculation of absolute atomic line strengths for many transitions in which either, as in the alkalis, the ionic core consists of closed shells, or, more generally, the energy differences of the ionic core levels may be ignored (Layzer and Garstang 1968, Weiss 1970). In such a calculation one uses single-channel energy eigenfunctions that are determined analytically for radial distances $r>r_{0}$, where $r_{0}$ is a cut-off radius beyond which it is assumed that there are no non-coulombic fields. The theory for such single- channel eigenfunctions has been developed by Ham (1955) and Seaton (1958) and is known as the Quantum Defect theory.

In most cases, however, neither the interactions between channels nor the energy differences of the ionic core levels can be ignored. As shown by Seaton (1966), the energy eigenfunctions in such multichannel problems may be represented in the region $r>r_{0}$ as a linear superposition of analytically known Coulomb functions. The coefficients of this superposition are related by Seaton (1966) to elements of the electron-ion scattering matrix. Recently Lu and Fano (Lu and Fano 1970, Fano 1970, Lu 1971) have shown that 
these coefficients, which form an eigenvector, may also be determined by semi-empirical analysis of a plot of Rydberg level energies. They have found that these eigenvectors may vary greatly along a series and from level to level of the same configuration (Fano 1970, Lu 1971). Using these eigenvectors and the Coulomb approximation one can calculate absolute line strengths for transitions between excited configurations. The Rydberg series to which the initial level belongs and that to which the final level belongs have to be analysed separately. The results of this analysis allow one to calculate line intensities for all transition arrays that differ by the principal quantum number of the initial level and/or the final level.

Using the method of Lu and Fano, we have analysed the two-channel neon series $2 \mathrm{p}^{5}\left({ }^{2} \mathrm{P}_{3 / 2,1 / 2}\right) n \mathrm{p} J=0$ and $2 \mathrm{p}^{5}\left({ }^{2} \mathrm{P}_{3 / 2,1 / 2}\right) n s J=1$ and have obtained for each series a set of five parameters that characterize the energy eigenvectors for all values of $n$. By using in addition the Coulomb approximation, we are thus able to calculate absolute intensities for any of the two-dimensional transition arrays $n \mathrm{p} J=0 \rightarrow n^{\prime} \mathrm{s} J=1$ and have done so for $n, n^{\prime}=3,4$. We note that for the particular array $3 p \rightarrow 3$ s our results are in good agreement with the recent accurate measurements of Bridges and Wiese (1970). This particular transition array has defied theoretical analysis for many years (eg Shortley 1935) until the recent calculations by Mehlhorn (1969) and Koenig (1971), who proceed by improving upon the intermediate coupling method. Both of these types of calculations, however, appear limited to transitions between low-lying levels (see eg Aymar et al 1970). We also compare our other calculated intensities with the compilation of Wiese et al (1966) and with the work of Murphy (1968).

Before presenting our analysis of neon, however, in the next section we first correct the formulas of $\mathrm{Lu}$ and Fano by inclusion of the energy dependent factors of Seaton (1966). These factors, which were left out by Lu and Fano, are necessary for our analysis of low-lying Rydberg levels. In order to give a self-contained treatment, we present all theoretical formulas necessary for calculating absolute intensities of transitions between multichannel Rydberg series. A step by step prescription for such calculations is also given. As in the work of Lu and Fano we restrict our consideration to systems in which only two ionic core levels need be considered.

\section{Theory}

The aim of the theory presented here is to extend the validity of Lu's formulas over an energy range encompassing low-lying Rydberg levels. The treatment of $\mathrm{Lu}$ assumed limiting values of the following functions defined by Seaton (1966):

$$
\begin{aligned}
& B(v, l)=v^{-2 l-2} \prod_{p=0}^{l}\left(v^{2}-p^{2}\right) \underset{v \rightarrow \infty}{\longrightarrow} 1 \\
& G(v, l)=\frac{B(v, l)}{2 \pi}\{\psi(v+l+1)+\psi(v-l)-2 \ln v\} \underset{v \rightarrow \infty}{\longrightarrow} 0 .
\end{aligned}
$$

In equation (2.1), $v$ and $l$ characterize an excited, but bound, electron in a Coulomb field; $v$ is the effective quantum number, which is related to the electron's energy (in atomic units) by the formula, $E=-1 / 2 v^{2} ; l$ is the electron's orbital angular momentum. The limiting values on the right in equation (2.1) obtain when the electron's energy is near threshold. 


\subsection{Coulomb basis functions}

Because we are considering the energy variation of the functions $B(v, l)$ and $G(v, l)$, we must distinguish two alternative pairs of Coulomb functions, each of which forms a basis for describing an arbitrary state of an electron in a Coulomb Field. These two pairs of Coulomb functions coincide in the energy region near threshold and hence were not distinguished by Lu. Following Fano (1970, Appendix B), we denote one of these pairs by $(f, g)$ and the other by $(\mathfrak{f}, \mathfrak{g})$. These functions are related by the following linear transformation:

$$
\begin{aligned}
\mathfrak{f}(v, l ; r) & =B^{1 / 2}(v, l) f(v, l ; r) \\
\mathfrak{g}(v, l ; r) & =B^{-1 / 2}(v, l)(g(v, l ; r)+G(v, l) f(v, l ; r))
\end{aligned}
$$

Near threshold the limiting values in equation (2.1) obtain and we see that the pairs of functions $(f, g)$ and $(\mathfrak{f}, g)$ coincide. The functions $f$ and $g$ are normalized to be energy independent for small radial distances $r$ (Seaton 1966). Their series expansions are given by Dehmer and Fano (1970). The pair $(\mathfrak{f}, \mathfrak{g})$ reduce for large radial distances to the expressions in equation (10) of Fano. They are a more convenient basis for representing energy eigenfunctions in the asymptotic region, since they are normalized per unit energy. They may be normalized per unit volume by a multiplicative factor (Fano 1970).

\subsection{Multichannel energy eigenfunctions}

The channels $i$ of a singly-excited atom, for particular values of total angular momentum $J$ and projection $M_{J}$, are identified by the state of the ionic core, the orbital angular momentum of the excited electron, and the coupling scheme of the combined ion electron system. We represent the ion wavefunction and its coupling with the angular and spin variables of the excited electron by the symbol $\Phi_{i}$. In the region $r>r_{0}$ we represent an arbitrary state of the combined electron-ion system quite generally by either of the alternative forms :

$$
\begin{aligned}
\Psi & =\frac{1}{r} \sum_{i} \Phi_{i}\left(\mathfrak{f}\left(v_{i}, l_{i} ; r\right) \mathfrak{c}_{i}-\mathfrak{g}\left(v_{i}, l_{i} ; r\right) \mathfrak{b}_{i}\right) \\
& =\frac{1}{r} \sum_{i} \Phi_{i}\left(f\left(v_{i}, l_{i} ; r\right) c_{i}-g\left(v_{i}, l_{i} ; r\right) d_{i}\right) .
\end{aligned}
$$

We concern ourselves in the rest of this section with obtaining coefficients $\left(\mathfrak{c}_{i}, \mathfrak{b}_{i}\right)$ or equivalently, $\left(c_{i}, d_{i}\right)$, appropriate to discrete excited states.

Interactions between channels $i$ occur in the core region, $r<r_{0}$ and may be described equivalently by either a scattering matrix $\mathbf{S}$ or a reaction matrix $\mathbf{R}$. Seaton (1966) has shown that these matrices may be written in terms of the known functions $B(v, l)$ and $G(v, l)$ and a product of unknown matrices $\mathbf{I} \mathbf{J}^{-1}$. By formally diagonalizing these matrices, Fano (Appendix B) and Lu have shown that one may represent the eigenstates of the $\mathbf{S}$ and $\mathbf{R}$ matrices by

$$
\Psi_{\beta}=\frac{1}{r} \sum_{i} \Phi_{i}\left(\mathfrak{f}\left(v_{i}, l_{i} ; r\right) \mathfrak{U}_{i \beta} \cos \left(\pi \mu_{\beta}\right)-\mathfrak{g}\left(v_{i}, l_{i} ; r\right) \mathfrak{U}_{i \beta} \sin \left(\pi \mu_{\beta}\right)\right)
$$

which is a special case of equation (2.3a). Similarly the eigenstates of the $\mathbf{I} \mathbf{J}^{-1}$ matrix 
may be represented by

$$
\Psi_{\alpha}=\frac{1}{r} \sum_{i} \Phi_{i}\left(f\left(v_{i}, l_{i} ; r\right) U_{i \alpha} \cos \left(\pi \xi_{\alpha}\right)-g\left(v_{i}, l_{i} ; r\right) U_{i \alpha} \sin \left(\pi \xi_{\alpha}\right)\right)
$$

In equation (2.4) the angle $\pi \mu_{\beta}\left(\pi \xi_{\alpha}\right)$ represents an eigenphase shift, and the matrix $\mathfrak{U}_{i \beta}\left(U_{i \alpha}\right)$ represents an orthogonal transformation that connects the eigenstates $\beta(\alpha)$ with the scattering channels $i$. In other words,

$$
R_{i j}=\sum_{\beta} \mathfrak{U}_{i \beta} \tan \left(\pi \mu_{\beta}\right) \tilde{\mathfrak{U}}_{j \beta}
$$

and

$$
\left(I J^{-1}\right)_{i j}=-\sum_{\alpha} U_{i \alpha} \cot \left(\pi \xi_{\alpha}\right) \tilde{U}_{j \alpha}
$$

The form of the coefficients $\left(c_{i}, b_{i}\right)$ and $\left(c_{i}, d_{i}\right)$ used in equation (2.3) may also be deduced by a phase-amplitude treatment (Dehmer and Fano 1970).

We now represent an arbitrary state of the electron-ion system as a linear superposition of one or the other of our newly defined sets of scattering eigenfunctions:

$$
\begin{aligned}
\Psi=\sum_{\beta} \Psi_{\beta} \mathscr{A}_{\beta} & =\frac{1}{r} \sum_{i} \Phi_{i}\left[\mathfrak{f}\left(v_{i}, l_{i} ; r\right) \sum_{\beta} \mathfrak{U}_{i \beta} \cos \left(\pi \mu_{\beta}\right) \mathscr{A}_{\beta}\right. \\
& \left.-\mathfrak{g}\left(v_{i}, l_{i} ; r\right)\left\{\sum_{\beta} \mathfrak{U}_{i \beta} \sin \left(\pi \mu_{\beta}\right) \mathscr{A}_{\beta}\right\}\right] \\
=\sum_{\alpha} \Psi_{\alpha} A_{\alpha} & =\frac{1}{r} \sum_{i} \Phi_{i}\left[f\left(v_{i}, l_{i} ; r\right)\left\{\sum_{\alpha} U_{i \alpha} \cos \left(\pi \xi_{\alpha}\right) A_{\alpha}\right\}\right. \\
& \left.-g\left(v_{i}, l_{i} ; r\right)\left\{\sum_{\alpha} U_{i \alpha} \sin \left(\pi \xi_{\alpha}\right) A_{\alpha}\right\}\right] .
\end{aligned}
$$

By substituting equation (2.2) in equation (2.6a) and equating the coefficients of $f$ and $g$ with those in equation ( $2.6 \mathrm{~b}$ ) one obtains the following relations between our alternative sets of coefficients :

$$
\begin{aligned}
A_{\alpha} & =\frac{1}{\sin \left(\pi \xi_{\alpha}\right)} \sum_{\beta}\left(\sum_{i} \tilde{U}_{i \alpha} B^{-1 / 2}\left(v_{i}, l_{i}\right) \mathfrak{U}_{i \beta}\right) \sin \left(\pi \mu_{\beta}\right) \mathscr{A}_{\beta} \\
\sum_{\beta} \tilde{\mathfrak{U}}_{i \beta} \cot \left(\pi \mu_{\beta}\right) \tilde{\mathfrak{U}}_{j \beta} & =B^{-1 / 2}\left(v_{i}, l_{i}\right)\left(\sum_{\alpha} U_{i \alpha} \cot \left(\pi \xi_{\alpha}\right) \tilde{U}_{j \alpha}+G\left(v_{i}, l_{i}\right) \delta_{i j}\right) B^{-1 / 2}\left(v_{j}, l_{j}\right)
\end{aligned}
$$

Recalling equation (2.5), note that equation (2.7b) is directly related to equations (4.13) . and (4.14) of Seaton (1966). Note also that near threshold when $B(v, l)$ and $G(v, l)$ assume their limiting values, equation (2.7) shows that the eigenstates $\alpha$ and $\beta$ become identical, that is $\mu_{\beta} \rightarrow \xi_{\alpha}, \mathfrak{U}_{i \beta} \rightarrow U_{i \alpha}$, and $\mathscr{A}_{\beta} \rightarrow A_{\alpha}$.

We have thus rewritten the wavefunction for an arbitrary state of the electron-ion system, given by equation (2.3), as a linear superposition of scattering eigenfunctions, given by equation (2.6). The alternative representations (2.6a) and (2.6b) serve the following purposes. Because the pair of Coulomb functions $(\mathfrak{f}, \mathfrak{g})$ are more appropriately normalized to describe the ion-electron system in the asymptotic region, equation (2.6a) will be used to represent $\Psi$ when we impose asymptotic boundary conditions. On the 
other hand, because the pair $(f, g)$ are nearly energy independent in the core region $r \approx r_{0}$, we expect the parameters $U_{i \alpha}$ and $\xi_{\alpha}$ (which determine the scattering eigenstates $\Psi_{\alpha}$ ) to be more slowly varying with energy than $\mathfrak{U}_{i \beta}$ and $\mu_{\beta}$. Indeed, the dependence of $\mathfrak{U}_{i \beta}$ and $\mu_{\beta}$ on energy is described largely by the functions $B(v, l)$ and $G(v, l)$, as exhibited by equation $(2.7 \mathrm{~b})$. We shall therefore fit the parameters $U_{i \alpha}$ and $\xi_{\alpha}$, rather than $\mathfrak{U}_{i \beta}$ and $\mu_{\beta}$, by an analysis of experimental data.

In order that equation (2.6a) may represent a discrete energy eigenstate, the right hand side must vanish at $r=\infty$. Using the asymptotic forms of $\mathfrak{f}$ and $\mathfrak{g}$ given by equation (10) of Fano (1970) we require the coefficients of certain rising exponentials to vanish, obtaining

$$
\sum_{\beta} \mathfrak{U}_{i \beta} \sin \left\{\pi\left(v_{i}+\mu_{\beta}\right)\right\} \mathscr{A}_{\beta}=0 \quad \text { for all } i
$$

which is the generalization of equation (2.18) of $\mathrm{Lu}(1971)$.

\subsection{Energy dependent Lu-Fano plots}

When only two ionic core levels are important for the analysis of a particular spectrum, equation (2.8) may be reduced further by separating the channels $i$ into two sets, P and Q, which are distinguished by the state of the ionic core. Since the effective quantum numbers $v_{i}$ depend only on the ionic core state, the set of $v_{i}$ contains only two distinct members, denoted $v_{\mathrm{P}}$ and $v_{\mathrm{Q}}$. Following Lu, we introduce a specific form for $\mathscr{A}_{\beta}$ which automatically satisfies equation (2.8) for each $i \in \mathrm{P}$,

$$
\mathscr{A}_{\beta}=\frac{1}{\sin \pi\left(v_{\mathrm{P}}+\mu_{\beta}\right)} \sum_{j \in \mathrm{Q}} \mathfrak{U}_{j \beta} \mathfrak{B}_{j}
$$

where the $\mathfrak{B}_{j}(j \in \mathrm{Q})$ are a new set of coefficients. We are left with the equations for $i \in \mathrm{Q}$ :

$$
\sum_{j \in \mathbf{Q}}\left\{\sum_{\beta} \mathfrak{U}_{i \beta} \frac{\sin \pi\left(v_{\mathrm{Q}}+\mu_{\beta}\right)}{\sin \pi\left(v_{\mathrm{P}}+\mu_{\beta}\right)} \tilde{\mathfrak{U}}_{j \beta}\right\} \mathfrak{B}_{j}=0 \quad i \in \mathrm{Q}
$$

which have non-trivial solutions provided

$$
\operatorname{det}\left|\sum_{\beta} \mathfrak{U}_{i \beta} \frac{\sin \pi\left(v_{\mathrm{Q}}+\mu_{\beta}\right)}{\sin \pi\left(v_{\mathbf{P}}+\mu_{\beta}\right)} \tilde{\mathfrak{U}}_{j \beta}\right|=0 \quad i, j \in \mathrm{Q} .
$$

For known $\mathfrak{U}_{i \beta}$ and $\mu_{\beta}$, equation (2.11) defines an implicit functional relation between $\mu \equiv-v_{\mathrm{P}}(\bmod 1)$ and $v_{\mathrm{Q}}(\bmod 1)$. We call the graph of $\mu \equiv-v_{\mathrm{P}}(\bmod 1)$ against $v_{\mathrm{Q}}(\bmod 1)$ obtained from equation (2.11) the energy dependent $\mathrm{Lu}-\mathrm{Fano}$ plot. It reduces to the ordinary Lu-Fano plot in the limit that $\mathfrak{H}_{i \beta} \rightarrow U_{i \alpha}$ and $\mu_{\beta} \rightarrow \xi_{\alpha}$.

Since the ordinary Lu-Fano plot is described in detail elsewhere ( $\mathrm{Lu}$ and Fano 1970), we shall only point out here how the energy dependent Lu-Fano plot differs from it. The energy dependent $\mathrm{Lu}-$ Fano plot is determined from equation (2.11), in which the parameters $\mu_{\beta}$ and $\mathfrak{U}_{i \beta}$ are assumed to vary with energy according to equation (2.7b). For this reason the energy dependent plot depends on the absolute values of $v_{\mathbf{P}}$ and $v_{\mathrm{Q}}$ rather than just on their values modulo 1 as in the ordinary plot. In practice the two plots are virtually identical except for small absolute values of $v_{\mathrm{P}}$ and $v_{\mathrm{Q}}$. As a result one finds that the ordinary Lu-Fano plot passes through the positions of all experimental energy levels (defined by experimental values of $v_{\mathrm{P}}$ and $v_{\mathrm{Q}}$ ) except the lowest 
energy ones. The energy dependent Lu-Fano plot, however, passes through even the lowest experimental energy levels.

An important result of the theory of Fano (1970) and Lu (1971) for our purposes is that the Lu-Fano plot intersects the diagonal line defined by $\mu\left(v_{\mathrm{Q}}\right)+v_{\mathrm{Q}}=1$ at the values $\mu\left(v_{\mathrm{Q}}\right)=\mu_{\beta}$. We thus expect any energy variation of the $\mu_{\beta}$ to cause the Lu-Fano plot to shift in a direction parallel to the diagonal. Using equation (2.7b) to describe the energy variation of the $\mu_{\beta}$ we may examine the energy dependence of the Lu-Fano plot in some detail. For $l_{i}>0$ the major influence on the energy dependence of the $\mu_{\beta}$ is that of the $B^{-1 / 2}\left(v_{i}, l_{i}\right)$ diagonal matrix, whose elements increase as $v_{i}$ decreases. Thus, in the approximation that we only consider the diagonal elements in equation (2.7b), $\mu_{\beta}$ increases or decreases as $v_{i}$ decreases depending on whether $\xi_{\alpha}$ is greater or less than $0 \cdot 5$. For $l_{i}=0, B\left(v_{i}, l_{i}\right)=1$ for all $v_{i}$ and $G\left(v_{i}, l_{i}\right)$ has only a very minor influence. For these s states the major energy variation is due to the $\xi_{\alpha}$, which we have assumed in this paper to vary linearly with energy. In the cases treated thus far, low-lying s electron levels are shifted up along the diagonal (ie to lower energies).

\subsection{Procedure for obtaining $\xi_{\alpha}$ and $U_{i \alpha}$ by fitting to experimental data}

We obtain the parameters $\xi_{\alpha}$ and $U_{i \alpha}$ by fitting equations (2.11) and (2.7b) to experimental energy level data. In the studies carried out so far we have found that the $\xi_{\alpha}$ vary slowly with energy. We represent this variation by the linear approximation $\xi_{\alpha}=\xi_{\alpha}^{0}+\xi_{\alpha}^{1} \epsilon$, where $\xi_{\alpha}^{0}$ and $\xi_{\alpha}^{1}$ are constants and where $\epsilon$ is the excited electron's energy with respect to the first ionization threshold. We represent the orthogonal matrix $U_{i \alpha}$ in terms of its independent parameters. A one-to-one correspondence between any orthogonal matrix and its expression in terms of generalized Euler angles has been given by Hoffman et al (1972). In our studies so far we have found these angles to be independent of energy. The procedure for obtaining all these parameters is:

(i) For each experimental term level $n$ compute $v_{\mathrm{P}}^{n}$ and $v_{\mathrm{Q}}^{n}$. Construct the plot of Lu and Fano (1970), and ignoring the lowest energy levels, connect the experimental points. The values of $\mu$ at which the resulting curves cross the diagonal $\mu+v_{\mathrm{Q}}=1$ give initial estimates of $\xi_{\alpha}^{0}$.

(ii) Assume initially that the $\xi_{\alpha}^{1}$ are zero.

(iii) Assume initially that the matrix $U_{i \alpha}$ is the $j j-L S$ angular momentum transformation matrix for the system in question ( $\mathrm{Lu} \mathrm{1971).} \mathrm{Such} \mathrm{matrices} \mathrm{have} \mathrm{been} \mathrm{tabulated} \mathrm{by}$ Kennedy and Cliff (1955). The formulas of Hoffman et al (1972) then allow one to obtain initial values for the generalized Euler angles.

(iv) Using initial estimates for the parameters given in (i) to (iii) we fit equations (2.11) and (2.7b) to experimental data by the following two-step procedure: First, for each experimental level $n$ we construct the matrix on the right in equation $(2.7 \mathrm{~b})$. Diagonalization of this matrix determines $\mu_{\beta}$ and $\mathfrak{U}_{i \beta}$ : the eigenvalues are $\cot \left(\pi \mu_{\beta}\right)$ and the eigenvectors are the columns of $\mathfrak{U}_{i \beta}$. Second, using the $\mu_{\beta}$ and $\mathfrak{H}_{i \beta}$ calculated at each level $n$ one constructs $\Sigma_{n} D_{n}^{2}$, where $D_{n}$ is the determinant in equation (2.11). A linearized regression procedure is then used to minimize $\Sigma_{n} D_{n}^{2}$ thereby obtaining successive improved estimates of the $\xi_{\alpha}^{0}$, the $\xi_{\alpha}^{1}$, and the generalized Euler angles of $U_{i \alpha}$.

(v) The linearized regression procedure outlined in (iv) is complicated by the number of transformations required between the sets of $\mu_{\beta}, \mathfrak{U}_{i \beta}$ and $\xi_{\alpha}, U_{i \alpha}$. One may avoid these transformations by rewriting equation (2.8) in terms of $\xi_{\alpha}, U_{i \alpha}$ and the functions $B\left(v_{i}, l_{i}\right)$ and $G\left(v_{i}, l_{i}\right)$, as is done in the Appendix. The resulting expression, however, is quite complicated. When $l_{i}$ is the same in all channels $i$ one may simplify this expression by 
introducing a renormalized effective quantum number, $v_{i}^{\mathrm{R}}$, defined by the relation

$$
\sin \pi v_{i}^{\mathrm{R}} \equiv \sin \pi v_{i}\left[\left(\sin \pi v_{i}\right)^{2}+\left\{\cos \pi v_{i} B\left(v_{i}, l_{i}\right)+\sin \pi v_{i} G\left(v_{i}, l_{i}\right)\right\}^{2}\right]^{-1 / 2}
$$

As shown in the Appendix, we may then write equation (2.8) as

$$
\sum_{\alpha} U_{i \alpha} \sin \pi\left(v_{i}^{\mathbf{R}}+\xi_{\alpha}\right) A_{\alpha}=0 \quad \text { for all } i .
$$

Just as (2.11) was derived from (2.8), we derive from (2.13) the relation:

$$
\operatorname{det}\left|\sum_{\alpha} U_{i \alpha} \frac{\sin \pi\left(v_{\mathrm{Q}}^{\mathrm{R}}+\xi_{\alpha}\right)}{\sin \pi\left(v_{\mathrm{P}}^{\mathrm{R}}+\xi_{\alpha}\right)} \tilde{U}_{j \alpha}\right|=0 \quad i, j \in Q .
$$

Equation (2.14) is used to obtain $\xi_{\alpha}$ and $U_{i \alpha}$ by fitting to experimental energy level data by a linearized regression procedure. No transformations between $\mu_{\beta}, \mathfrak{U}_{i \beta}$ and $\xi_{\alpha}, U_{i \alpha}$ are necessary. Note that the $v_{i}^{\mathrm{R}}$ all lie on the energy independent Lu-Fano plot, even for low-lying levels (except for the slight shifts due to the energy dependence of the $\xi_{\alpha}$ ).

Note that equations (2.11) and (2.7b) are not in general sufficient to determine the orthogonal matrix $U_{i \alpha}$ completely. This is because the indices i and $j$ in equation (2.11) are restricted to $(i, j) \in \mathrm{Q}$ and hence in general not all the generalized Euler angles on which $\mathfrak{U}_{i \beta}$ depends appear in equation (2.11). In such cases we must include an additional equation with $(i, j) \in \mathrm{P}$ in the linearized regression procedure of step (iv). This equation is obtained by noting that instead of writing $\mathscr{A}_{\beta}$ as in equation (2.9) we might alternatively have written it as

$$
\mathscr{A}_{\beta}=\frac{1}{\sin \pi\left(v_{\mathrm{Q}}+\mu_{\beta}\right)} \sum_{j \in \mathrm{P}} \tilde{\mathfrak{U}}_{j \beta} \mathfrak{B}_{j}
$$

where these $\mathfrak{B}_{j}(j \in \mathrm{P})$ are different from those of (2.9). Just as equation (2.11) was derived from (2.9) we obtain from $\left(2.9^{\prime}\right)$ the following equation:

$$
\operatorname{det}\left|\mathfrak{U}_{i \beta} \frac{\sin \pi\left(v_{\mathrm{P}}+\mu_{\beta}\right)}{\sin \pi\left(v_{\mathrm{Q}}+\mu_{\beta}\right)} \tilde{\mathfrak{U}}_{j \beta}\right|=0 \quad i, j \in \mathrm{P} .
$$

Thus all these angles may be obtained by fitting equations (2.11), (2.11'), and (2.7b) to experimental energy level data. For the calculations presented in this paper, however, equations (2.11) and (2.7b) suffice to determine all needed parameters.

\subsection{Formulas for normalized bound-state eigenfunctions and dipole matrix elements}

Having determined the parameters $\xi_{\alpha}^{0}, \xi_{\alpha}^{1}$ and $U_{i \alpha}$ by the methods outlined above, we calculate the set of eigenvector coefficients $\mathscr{A}_{\beta}^{n}$ and $A_{\alpha}^{n}$ (up to a normalization factor) for each level $n$ from equations (2.10), (2.9), and (2.7). (In the special case when all $l_{i}$ are equal we calculate $A_{\alpha}^{n}$ directly from (2.13).) Using the form for the wavefunction given in equation (2.6a), we obtain the normalization constant by the method described in section VI B of Fano:

$\int\left|\Psi_{n}\right|^{2} \mathrm{~d} \tau=\sum_{i}\left(v_{i}^{n}\right)^{3}\left(\sum_{\beta} \mathfrak{U}_{i \beta} \cos \pi\left(v_{i}^{n}+\mu_{\beta}\right) \mathscr{A}_{\beta}^{n}\right)\left(\sum_{\beta^{\prime}} \mathfrak{U}_{i \beta^{\prime}} \cos \pi\left(v_{i}^{n}+\mu_{\beta^{\prime}}\right) \mathscr{A}_{\beta^{\prime}}^{n} \zeta_{i \beta^{\prime}}\right)$

where

$$
\zeta_{i \beta}=1+\frac{\mathrm{d} \mu_{\beta}}{\mathrm{d} v_{i}}
$$


Note that in the paper by $\mathrm{Lu} \zeta_{i \beta}$ is assumed to be equal to unity. In this paper $\zeta_{i \beta}$ was calculated numerically by using equation (2.7b) to generate values of $\mu_{\beta}$ at successively closer values of $v_{i}$.

Having completely defined the bound energy eigenstates in equation (2.6) we now transform them to a form that is more convenient for the numerical applications of this paper. We started with the form given in equation (2.6) because we wished to make connection with the theory of $\mathrm{Lu}$, which was developed for application to a wider range of phenomena. Using equation (32) of Seaton (1958) we rewrite the function $g\left(v_{i}, l_{i} ; r\right)$ as :

$g\left(v_{i}, l_{i} ; r\right)=-\left\{B\left(v_{i}, l_{i}\right) \cot \pi v_{i}+G\left(v_{i}, l_{i}\right)\right\} f\left(v_{i}, l_{i} ; r\right)-\frac{\Gamma\left(l_{i}+1-v_{i}\right)}{\pi\left(v_{i}\right)^{l_{i}}} \theta\left(v_{i}, l_{i} ; r\right)$

where $\theta\left(v_{i}, l_{i} ; r\right)$ is just the Whittaker function $W_{v, l+1 / 2}(2 r / v)$, which decreases exponentially as $r$ becomes large. Substituting the expressions for $\mathfrak{f}$ and $\mathfrak{g}$ given by equation (2.2) in equation (2.6a) and replacing the function $g$ by equation (2.17) we obtain:

$$
\begin{aligned}
\Psi_{n} & =\frac{1}{r} \sum_{i} \Phi_{i} \theta\left(v_{i}^{n}, l_{i} ; r\right) \frac{\Gamma\left(l_{i}+1-v_{i}^{n}\right)}{\pi\left(v_{i}^{n}\right)^{l_{i}}} B^{-1 / 2}\left(v_{i}^{n}, l_{i}\right)\left(\sum_{\beta} \mathfrak{U}_{i \beta} \sin \pi \mu_{\beta} \mathscr{A}_{\beta}^{n}\right) \\
& =\frac{1}{r} \sum_{i} \Phi_{i} \theta\left(v_{i}^{n}, l_{i} ; r\right) \frac{\Gamma\left(l_{i}+1-v_{i}^{n}\right)}{\pi\left(v_{i}^{n}\right)^{l_{i}}}\left(\sum_{\alpha} U_{i \alpha} \sin \pi \xi_{\alpha} A_{\alpha}^{n}\right) .
\end{aligned}
$$

In deriving equation (2.18) we have made use of both the boundary condition on bound states, given by equation (2.8), and the transformation between our different sets of coefficients, given by equation (2.7). Equation (2.18b) shows that the parameters $\xi_{\alpha}$ and $U_{i \alpha}$ determine the bound state energy eigenfunctions for every member $n$ of the multichannel Rydberg series.

In the next section we analyse a pair of two-channel Rydberg series in neon, which we designate here simply by a and b. For each series we obtain parameters $\xi_{\alpha}$ and $U_{i \alpha}$. The $q$ th component of the dipole matrix element for a transition between level $n$ of Rydberg series a and level $m$ of Rydberg series b is calculated as follows, using wavefunctions of the form given by equation $(2.18 \mathrm{~b})$ :

$$
\left\langle\Psi_{n}^{\mathrm{a}}\left|\sum_{i} r_{i} C_{q}^{[1]}\left(\Omega_{i}\right)\right| \Psi_{m}^{\mathrm{b}}\right\rangle=\sum_{i, j} D_{i j}^{n m} Y_{i j}^{\mathrm{ab}} C_{i}^{\mathrm{an}} C_{j}^{\mathrm{b} m}
$$

where

$$
\begin{aligned}
D_{i j}^{n m} & \equiv \frac{\Gamma\left(l_{i}+1-v_{i}^{n}\right) \Gamma\left(l_{j}+1-v_{j}^{m}\right)}{\pi^{2}\left(v_{i}^{n}\right)^{l_{i}}\left(v_{j}^{m}\right)^{l_{j}}}\left(\int_{r_{0}}^{\infty} \mathrm{d} r \theta\left(v_{i}^{n}, l_{i} ; r\right) r \theta\left(v_{j}^{m}, l_{j} ; r\right)\right) \\
Y_{i j}^{\mathrm{ab}} & \equiv\left\langle\Phi_{i}^{\mathrm{a}}\left|C_{q}^{[1]}(\Omega)\right| \Phi_{j}^{\mathrm{b}}\right\rangle
\end{aligned}
$$

(where $\Omega$ are the angular variables of the excited electron),

$$
\begin{aligned}
C_{i}^{\mathrm{a} n} & \equiv \sum_{\alpha} U_{i \alpha}^{\mathrm{a}} \sin \left(\pi \xi_{\alpha}^{\mathrm{a}}\right) A_{\alpha}^{n} \\
C_{j}^{\mathrm{b} m} & \equiv \sum_{\alpha^{\prime}} U_{j \alpha^{\prime}}^{\mathrm{b}} \sin \left(\pi \xi_{\alpha^{\prime}}^{\mathrm{b}}\right) A_{\alpha^{\prime}}^{m} .
\end{aligned}
$$

The matrix element of $C_{q}^{[1]}(\Omega)$ has been left in abstract form since it is easier to evaluate it in each individual case by standard methods (see eg Briggs 1971). The line strengths presented in the next section were obtained by squaring equation (2.19) and summing 
over $q$ and all initial and final magnetic quantum numbers. Note that in calculating the radial matrix elements in equation $(2.20 \mathrm{a})$ we have employed the Coulomb approximation.

\section{Application to line strengths in neon}

For each of the neon two-channel Rydberg series $2 \mathrm{p}^{5}\left({ }^{2} \mathrm{P}_{3 / 2,1 / 2}\right) n \mathrm{~s} J=1$ and $2 \mathrm{p}^{5}\left({ }^{2} \mathrm{P}_{3 / 2,1 / 2}\right) n^{\prime} \mathrm{p} J=0$ we have determined a set of five parameters which characterize the energy eigenvectors for each $n, n^{\prime}$. In analysing the $n \mathrm{~s} J=1$ series we have ignored their interaction with the $n \mathrm{~d} J=1$ series since a Lu-Fano plot of the $n \mathrm{~s} J=1$ and $n d J=1$ energy levels shows no indication of any interaction. This is unlike the the situation in xenon treated by $\mathrm{Lu}$ (1971). In this section we present line strengths for transitions between the $n \mathrm{~s} J=1$ and $n^{\prime} \mathrm{p} J=0$ series for values $n, n^{\prime}=3,4$. The parameters listed below may be used by other workers to obtain additional line strengths. Experimental term levels for neon were obtained from Moore (1949) and experimental ionization limits from Moore (1970).

\subsection{Tests for the applicability of the Coulomb approximation}

Our eigenfunctions are, of course, determined only in the region $r>r_{0}$. Hence it is necessary to our calculations that we may ignore the contribution of the region $0 \leqslant r \leqslant r_{0}$ to the radial integrals in equation (2.20a). To test this contribution we have computed radial dipole matrix elements for the transitions of interest in this paper using the central field wavefunctions of Herman and Skillman (1963). We have computed each integral over the two ranges $0 \leqslant r \leqslant r_{0}$ and $0 \leqslant r \leqslant \infty$ and compared the results, which are listed in table 1 . The value of $r_{0}$ is that given by Herman and Skillman;

Table 1. Test of Coulomb approximation for neon using Herman-Skillman wavefunctions and $r_{0}=1.67$ Bohr.

\begin{tabular}{lcll}
\hline A & B & C & D \\
\hline $3 s \rightarrow 3 p$ & -4.909 & $-0.874 \times 10^{-2}$ & 0.18 \\
$3 s \rightarrow 4 p$ & -0.327 & $-0.520 \times 10^{-2}$ & 1.59 \\
$4 s \rightarrow 3 p$ & +4.498 & $-0.435 \times 10^{-2}$ & 0.10 \\
$4 s \rightarrow 4 p$ & -11.133 & $-0.261 \times 10^{-2}$ & 0.02 \\
\hline
\end{tabular}

\footnotetext{
A, dipole transition.

$\mathrm{B}, \mathrm{C}$, radial dipole matrix element integrated over the ranges $0 \leqslant r \leqslant \infty$ and $0 \leqslant r \leqslant r_{0}$ respectively (atomic units).

$\mathrm{D}$, absolute value of the ratio $\mathrm{C}$ to $\mathrm{B}$ in per cent.
}

we shall use this value for $r_{0}$ in all the other calculations presented in this paper. From the results presented in table 1 we see that, except for the transition $3 \mathrm{~s} \rightarrow 4 \mathrm{p}$, the use of the Coulomb approximation should have negligible effect on the accuracy of the computed line strengths. Even in the case of $3 s \rightarrow 4 p$ line strengths, the Coulomb Approximation should introduce inaccuracies of no more than a few per cent.

Alternatively, one may test the Coulomb approximation by computing line intensities for transitions between one-channel Rydberg series, which occur when the coupling 
of one of the core levels with the excited electron for specified total $J$ is incompatible with angular momentum selection rules. The formulas of $\S 2$ simplify for one-channel problems as follows: equation (2.7b), which relates $\mu_{\beta}$ and $\xi_{\alpha}$, becomes,

$$
\cot \pi \mu_{\alpha}=B^{-1}(v, l)\left(\cot \pi \xi_{\alpha}+G(v, l)\right)
$$

equation (2.8), which is the necessary condition for bound states, becomes,

$$
\sin \pi\left(v+\mu_{\alpha}\right)=0
$$

and, finally, equation (2.18a) for the normalized bound state energy eigenfunction becomes :

$$
\Psi_{n}=\frac{1}{r} \Phi \theta(v, l ; r) \frac{\Gamma\left(l+1-v^{n}\right)}{\pi\left(v^{n}\right)^{l}} \frac{B^{-1 / 2}(v, l) \sin \pi \mu_{\alpha}}{\left(v^{n}\right)^{3 / 2} \zeta_{\alpha}^{1 / 2}} .
$$

In the above equation $\alpha$ denotes the $L S$-coupling term identifying the one-channel series. By using experimental values for $v^{n}$ and computing $\mu_{\alpha}$ from equation (3.2) for each value of $v^{n}$, the eigenfunction in equation (3.3) is completely determined. Thus the accuracy of line strengths computed using one-channel eigenfunctions depends largely on the validity of the Coulomb approximation.

For this reason we have computed line strengths for transitions between the neon one-channel series $2 \mathrm{p}^{5}\left({ }^{2} \mathrm{P}_{3 / 2}\right) n s\left({ }^{3} \mathrm{P}_{2}\right)$ and $2 \mathrm{p}^{5}\left({ }^{2} \mathrm{P}_{3 / 2}\right) n^{\prime} \mathrm{p}\left({ }^{3} \mathrm{D}_{3}\right)$ for $n, n^{\prime}=3,4$. The results are presented in table 2. Bridges and Wiese (1970) have determined experimentally the

\begin{tabular}{|c|c|c|c|c|c|c|}
\hline \multirow[b]{2}{*}{ Transition } & \multicolumn{6}{|c|}{ Line strengths (atomic units) } \\
\hline & $\begin{array}{l}\text { Present } \\
\text { work }\end{array}$ & $\begin{array}{l}\text { Bridges and } \\
\text { Wiese (1970) }\end{array}$ & $\begin{array}{l}\text { Koenig } \\
(1971)\end{array}$ & $\begin{array}{l}\text { Mehlhorn } \\
\text { (1969) }\end{array}$ & $\begin{array}{l}\text { Murphy } \\
\text { (1968) }\end{array}$ & $\begin{array}{l}\text { Wiese et al } \\
(1966)\end{array}$ \\
\hline $3 s \rightarrow 3 p\left(1 s_{5} \rightarrow 2 p_{9}\right)$ & 47.2 & $45 \cdot 9 \pm 3 \cdot 2$ & 48.9 & 45.9 & & \\
\hline $3 \mathrm{~s} \rightarrow 4 \mathrm{p}\left(1 \mathrm{~s}_{5} \rightarrow 3 \mathrm{p}_{9}\right)$ & 0.270 & & & & & $1.44 \pm 0.36$ \\
\hline $4 \mathrm{~s} \rightarrow 3 \mathrm{p}\left(2 \mathrm{~s}_{5} \rightarrow 2 \mathrm{p}_{9}\right)$ & 42.4 & & & & $42 \cdot 3$ & \\
\hline $4 s \rightarrow 4 p\left(2 s_{5} \rightarrow 3 p_{9}\right)$ & 262 & & & & 262 & $260 \pm 130$ \\
\hline
\end{tabular}

Table 2. Line strengths for transitions between the one-channel neon series $2 \mathrm{p}^{5}\left({ }^{2} \mathrm{P}_{3 / 2}\right) n s\left({ }^{3} \mathrm{P}_{2}\right)$ $2 \mathrm{p}^{5}\left({ }^{2} \mathrm{P}_{3 / 2}\right) n \mathrm{p}\left({ }^{3} \mathrm{D}_{3}\right)$

line strength for the $3 \mathrm{~s} J=2 \rightarrow 3 \mathrm{p} J=3$ transition (denoted $1 \mathrm{~s}_{5} \rightarrow 2 \mathrm{p}_{9}$ in Paschen notation) to within $\pm 7 \%$. Our calculated line strength agrees very well with their experimental value thus further confirming the validity of the Coulomb approximation for $3 s \rightarrow 3 p$ transitions. Our result also agrees with the calculations of Koenig (1971) and Mehlhorn (1969), who use a single value of the radial dipole matrix element for the whole transition array $3 \mathrm{~s} \rightarrow 3 \mathrm{p}$.

A value of the line strength for the transition $3 \mathrm{~s} J=2 \rightarrow 4 \mathrm{p} J=3$ determined from the lifetime data of Klose (1966) for the $4 \mathrm{p}$ level is included in the compilation by Wiese et al (1966). This line strength is not expected to very accurate (Wiese et al 1966). Our calculated result differs from it by a factor of five and in view of the small expected inaccuracies arising from the Coulomb Approximation (see table 1) we suspect the accuracy of the experimental line strength.

Despite the lack of experimental results for comparison, we have also calculated line strengths for the transitions $4 \mathrm{~s} J=2 \rightarrow n \mathrm{p} J=3$ for $n=3,4$. Our results agree well 
with the intermediate coupling calculations of Murphy (1968) and Ufford (1937), the latter being included in Wiese et al. This agreement is expected since intermediate coupling calculations are put on an absolute basis by using the Coulomb approximation.

\subsection{Analysis of two-channel Rydberg series}

We now discuss briefly our analysis of the neon two-channel series $2 \mathrm{p}^{5}\left({ }^{2} \mathrm{P}_{3 / 2,1 / 2}\right) n s J=1$ and $2 \mathrm{p}^{5}\left({ }^{2} \mathrm{P}_{3 / 2,1 / 2}\right) n \mathrm{p} J=0$. For each of these two series the matrix $U_{i \alpha}$ is a $2 \times 2$ orthogonal matrix which depends on a single angle $\phi$ :

$$
U=\left(\begin{array}{rr}
\cos \phi & \sin \phi \\
-\sin \varphi & \cos \varphi
\end{array}\right) .
$$

Whereas the scattering channels $i$ are defined to be $j j$-coupled, the exact character of the eigenstates $\alpha$ depends on the value of $\varphi$ obtained from fitting to experimental data. However, since the eigenstates $\alpha$ are determined by interactions in the region $r<r_{0}$, in which electrostatic interactions are usually stronger than spin-orbit interactions, we expect the eigenstates $\alpha$ in general to tend towards $L S$-coupling. Furthermore, we know that the spin-orbit interaction in neon is weak so that we expect the eigenstates $\alpha$ to be closer to $L S$-coupling than is usual. Our initial estimate for $\varphi$ is $\varphi_{j j-L S}$, which is the angle defining the $j j-L S$ angular transformation matrix. In what follows we shall identify the states $\alpha$ by their predominant $L S$ coupling character, for example $\alpha=10$ implies that $L=1$ and $S=0$.

We note two further points of our fitting procedure. First, we allowed the angle $\varphi$ to vary linearly with energy, ie $\varphi=\varphi^{0}+\varphi^{1} \epsilon$, where $\varphi^{0}$ and $\varphi^{1}$ are constants. However, for the series analysed below we found $\varphi^{1}$ to be less than 0.0001 au and hence we ignored it. Second, we found it helpful to use the approximate $J$ independence of the eigenstates $\alpha$ and of the parameters $\xi_{\alpha}^{0}$ and $\xi_{\alpha}^{1}$ (see Lu 1971, Appendix). Specifically, the eigenstate $\alpha$ characterized by the $L S$-coupling term ${ }^{3} \mathrm{P}$ (ie, $\alpha=11$ ) occurs in three of the neon $2 \mathrm{p}^{5} n \mathrm{~s}$ Rydberg series: it occurs not only in the two-channel $n \mathrm{~s} J=1$ series of interest below, but also in the one-channel series $n s J=0$ and $n s J=2$. The values of $\xi_{11}^{0}$ and $\xi_{11}^{1}$ obtained from analysis of each of the one-channel series are given in table 3 . These values were used as initial estimates in our fitting of the two-channel $n s J=1$ series and turned out to be quite close to the parameters $\xi_{11}^{0}$ and $\xi_{11}^{1}$ obtained for the twochannel series (see table 4). Since one-channel Rydberg series are easier to analyse than

Table 3. Scattering parameters for the neon one-channel Rydberg series $2 \mathrm{p}^{5}\left({ }^{2} \mathrm{P}_{3 / 2}\right) n s\left({ }^{3} \mathrm{P}_{2}\right)$ and $2 \mathrm{p}^{5}\left({ }^{2} \mathrm{P}_{1 / 2}\right) n s\left({ }^{3} \mathrm{P}_{0}\right)$

\begin{tabular}{lll}
\hline Series & $\xi_{11}^{0}$ & $\xi_{11}^{1}$ \\
\hline $2 \mathrm{p}^{5}\left({ }^{2} \mathrm{P}_{3 / 2}\right) n s\left({ }^{3} \mathrm{P}_{2}\right)$ & 0.3142 & -0.1369 \\
$2 \mathrm{p}^{5}\left({ }^{2} \mathrm{P}_{1 / 2}\right) n s\left({ }^{3} \mathrm{P}_{0}\right)$ & 0.3149 & -0.1359 \\
\hline
\end{tabular}

Table 4. Scattering parameters for the two-channel neon series $2 \mathrm{p}^{5}\left({ }^{2} \mathrm{P}_{3 / 2,1 / 2}\right) n s J=1$.

\begin{tabular}{rr}
\hline$\phi=0.6150$ & $\left(\phi_{\mathrm{jj}-L S}=0.6155\right)$ \\
$\xi_{10}^{0}=0.2832$ & $\xi_{10}^{1}=-0.1372$ \\
$\xi_{11}^{0}=0.3151$ & $\xi_{11}^{1}=-0.1348$ \\
\hline
\end{tabular}




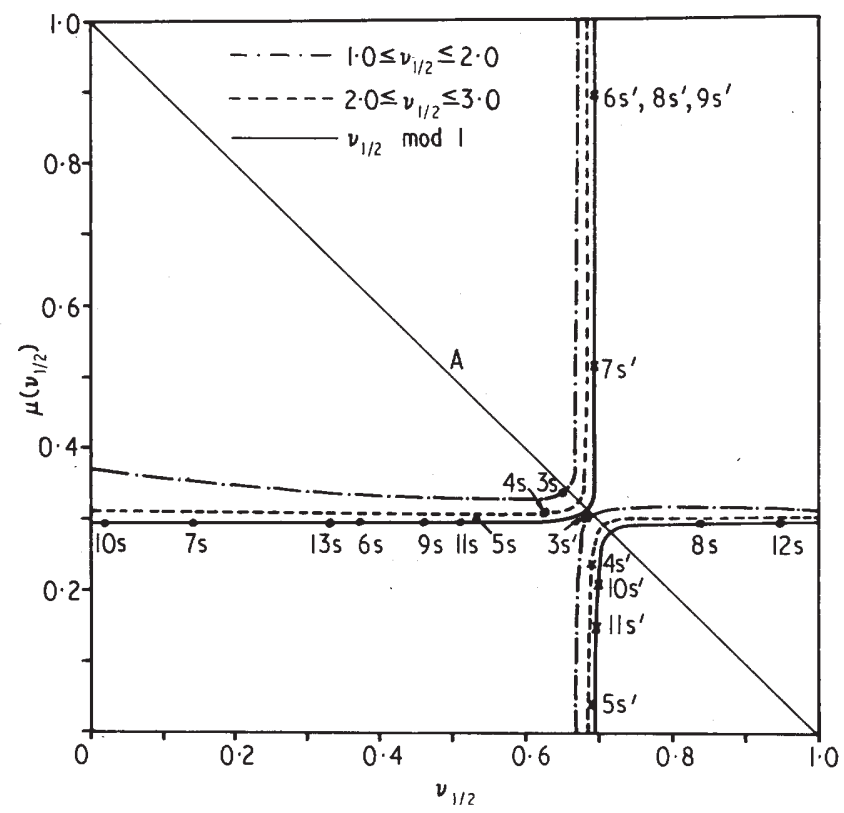

Figure 1. Energy dependent Lu-Fano plot for the neon series $2 \mathrm{p}^{5}\left({ }^{2} \mathrm{P}_{3 / 2}\right) n s J=1$ (solid circles) and $2 \mathrm{p}^{5}\left({ }^{2} \mathrm{P}_{1 / 2}\right) n \mathrm{~s}^{\prime} J=1$ (crosses). - - - . and - - - f first two cycles of the curve; energy independent Lu-Fano plot (applicable near threshold).

Curve $\mathrm{A} \mu\left(v_{1 / 2}\right)+v_{1 / 2}=1$.

Table 5. Scattering parameters for the two-channel neon series $2 \mathrm{p}^{5}\left({ }^{2} \mathrm{P}_{3 / 2,1 / 2}\right) n \mathrm{p} J=0$.

\begin{tabular}{rlrl}
\hline$\phi$ & $=-0.6097$ & $\left(\varphi_{\mathrm{j} j-L S}\right.$ & $=-0.6155)$ \\
$\xi_{00}^{0}$ & $=0.6783$ & $\xi_{00}^{1}$ & $=-0.0375$ \\
$\xi_{11}^{0}$ & $=0.8091$ & $\xi_{11}^{1}$ & $=0.1237$ \\
\hline
\end{tabular}

multichannel ones, it is advantageous to exploit the approximate $J$ independence of the $\alpha$ eigenstates whenever possible.

The parameters obtained for the neon two-channel series $2 \mathrm{p}^{5}\left({ }^{2} \mathrm{P}_{3 / 2,1 / 2}\right) n s J=1$ and $2 \mathrm{p}^{5}\left({ }^{2} \mathrm{P}_{3 / 2,1 / 2}\right) n \mathrm{p} J=0$ are listed in tables 4 and 5 . We see that, as expected, the angle $\varphi$ is quite close to $\varphi_{j j-L S}$ in each case. Using these parameters we have plotted in figure 1 and figure 2 the first two cycles of the energy dependent Lu-Fano plot. (In these two figures we have written $v_{\mathrm{Q}}$ as $v_{1 / 2}$, where the subscript $1 / 2$ refers to the upper ionic core level, ${ }^{2} \mathrm{P}_{1 / 2}$.) We note that these lowest two cycles pass through the positions of the lowest energy levels of each series. The solid line in each figure is the ordinary Lu-Fano plot, to which the energy dependent plot tends after many cycles. (Note that we have not explicitly drawn all these cycles). We see that the higher experimental energy levels all lie on this line.

These neon two-channel series have also been analysed by Saraph and Seaton (1971), who use calculated electron-ion scattering matrices to predict energy level positions. Our energy dependent Lu-Fano plots predict position coordinates $\left(\mu^{n}, v_{1 / 2}^{n}\right)$ for each level $n$ which are no more than a distance of \pm 0.005 units from the experimental positions (except for $8 \mathrm{p}$ in figure 2). This accuracy compares favourably with that of Saraph and Seaton for these series. 


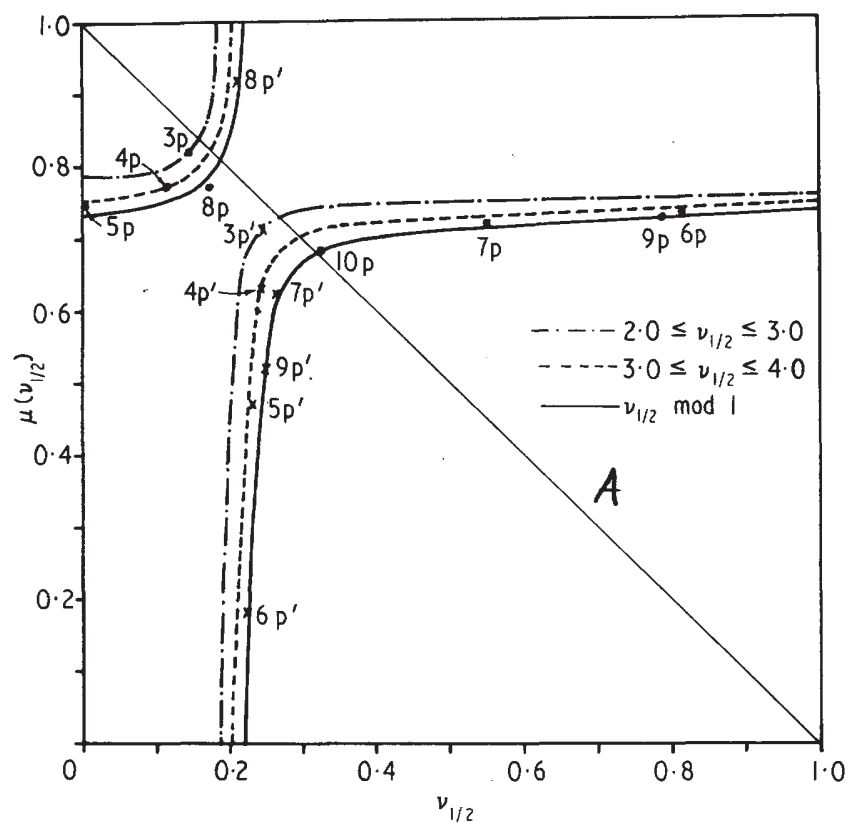

Figure 2. Energy dependent Lu-Fano plot for the neon series $2 \mathrm{p}^{5}\left({ }^{2} \mathrm{P}_{3 / 2}\right) n \mathrm{p} J=0$ (solid circles) and $2 \mathrm{p}^{5}\left({ }^{2} \mathrm{P}_{1 / 2}\right) n \mathrm{p}^{\prime} J=0$ (crosses). - - - - and - - - first two cycles of the curve; energy independent Lu-Fano plot (applicable near threshold).

Curve A $\mu\left(v_{1 / 2}\right)+v_{1 / 2}=1$.

3.3. Line strengths for transitions between the series $2 p^{5}\left({ }^{2} P_{3 / 2,1 / 2}\right) n s J=1$ and $2 p^{5}\left({ }^{2} P_{3 / 2,1 / 2}\right) n^{\prime} \mathrm{p} J=0$ for $n, n^{\prime}=3,4$

Using the parameters in tables 4 and 5 and the formulas of $\S 2.5$ we computed line strengths for transitions between the series analysed above. The results are given in table 6, where, except for the results quoted by Wiese et al (1966), we have converted other authors' transition probabilities to line strengths.

Before discussing each transition in detail, we wish to point out the differences between our results and those obtained from an intermediate coupling (IC) calculation. IC theory assumes that $n$ and $l$ are good quantum numbers and that the radial integrals (ie $D_{i j}^{n m}$ in equation (2.20a)) depend only on initial and final values of $n$ and $l$. Quantum defect theory, however, does not make these assumptions. Thus the agreement between our results and those of IC calculations depends on how well the assumptions of IC theory are met. Our results show that they are well met in some cases, but not in others. To be specific, the assumptions of IC theory lead to the following results, which are not true generally: (i) orthogonality of the coefficient vectors $C^{\mathrm{bm}}$ (with components defined by equation (2.20d)) belonging to levels $m$ of the same configuration; (ii) the $J$-file sum rule of Shortley (1935). Result (i) leads to the IC prediction that line strengths for $s_{4} \rightarrow p_{3}$ and $s_{2} \rightarrow p_{1}$ transitions (Paschen notation) should be equal and that line strengths for $s_{4} \rightarrow p_{1}$ and $s_{2} \rightarrow p_{3}$ transitions should be equal. Our line strengths in table 6 show that these IC predictions are not always valid. The $J$-file sum rule predicts that the entries along each row in table 7 should be equal. We have employed our line strengths in table 7 and we see once more that the IC predictions are not always valid.

We now discuss each of our computed transitions in detail. 
Table 6. Line strengths for transitions between the two-channel neon series $2 \mathrm{p}^{5}\left({ }^{2} \mathrm{P}_{3 / 2,1 / 2}\right) n \mathrm{~s}$ $J=1$ and $2 \mathrm{p}^{5}\left({ }^{2} \mathrm{P}_{3 / 2,1 / 2}\right) n \mathrm{p} J=0$

\begin{tabular}{|c|c|c|c|c|c|c|}
\hline \multirow[t]{2}{*}{ Transition } & \multicolumn{6}{|c|}{ Line strengths (atomic units) } \\
\hline & $\begin{array}{l}\text { Present } \\
\text { work }\end{array}$ & $\begin{array}{l}\text { Bridges and } \\
\text { Wiese }(1970)\end{array}$ & $\begin{array}{l}\text { Keonig } \\
\text { (1971) }\end{array}$ & $\begin{array}{l}\text { Mehlhorn } \\
(1969)\end{array}$ & $\begin{array}{l}\text { Murphy } \\
(1968)\end{array}$ & $\begin{array}{l}\text { Wiese et al } \\
(1966)\end{array}$ \\
\hline $3 \mathrm{~s} \rightarrow 3 \mathrm{p}\left(1 \mathrm{~s}_{4} \rightarrow 2 \mathrm{p}_{3}\right)$ & $6 \cdot 72$ & $6.45 \pm 0.45$ & $6 \cdot 61$ & $6 \cdot 53$ & & \\
\hline $3 \mathrm{~s} \rightarrow 3 \mathrm{p}^{\prime}\left(1 \mathrm{~s}_{4} \rightarrow 2 \mathrm{p}_{1}\right)$ & 0.077 & $0.070 \pm 0.011$ & 0.068 & 0.036 & & \\
\hline $3 \mathrm{~s}^{\prime} \rightarrow 3 \mathrm{p}\left(1 \mathrm{~s}_{2} \rightarrow 2 \mathrm{p}_{3}\right)$ & 0.027 & $0.049 \pm 0.007$ & 0.044 & 0.036 & & \\
\hline $3 \mathrm{~s}^{\prime} \rightarrow 3 \mathrm{p}^{\prime}\left(1 \mathrm{~s}_{2} \rightarrow 2 \mathrm{p}_{1}\right)$ & 6.92 & $6.98 \pm 0.49$ & $7 \cdot 16$ & 6.53 & & \\
\hline $3 \mathrm{~s} \rightarrow 4 \mathrm{p}\left(1 \mathrm{~s}_{4} \rightarrow 3 \mathrm{p}_{3}\right)$ & 0.087 & & & & & $0.173 \pm 0.043$ \\
\hline $3 \mathrm{~s} \rightarrow 4 \mathrm{p}^{\prime}\left(1 \mathrm{~s}_{4} \rightarrow 3 \mathrm{p}_{1}\right)$ & $0.2 \times 10^{-3}$ & & & & & \\
\hline $3 \mathrm{~s}^{\prime} \rightarrow 4 \mathrm{p}\left(1 \mathrm{~s}_{2} \rightarrow 3 \mathrm{p}_{3}\right)$ & 0.023 & & & & & \\
\hline $3 s^{\prime} \rightarrow 4 p^{\prime}\left(1 s_{2} \rightarrow 3 p_{1}\right)$ & $0 \cdot 182$ & & & & & $0.156 \pm 0.039$ \\
\hline $4 \mathrm{~s} \rightarrow 3 \mathrm{p}\left(2 \mathrm{~s}_{4} \rightarrow 2 \mathrm{p}_{3}\right)$ & 5.48 & & & & $4 \cdot 20$ & \\
\hline $4 s \rightarrow 3 p^{\prime}\left(2 s_{4} \rightarrow 2 p_{1}\right)$ & 3.66 & & & & 1.86 & \\
\hline $4 \mathrm{~s}^{\prime} \rightarrow 3 \mathrm{p}\left(2 \mathrm{~s}_{2} \rightarrow 2 \mathrm{p}_{3}\right)$ & 1.69 & & & & $1 \cdot 86$ & \\
\hline $4 s^{\prime} \rightarrow 3 p^{\prime}\left(2 s_{2} \rightarrow 2 p_{1}\right)$ & 7.65 & & & & $4 \cdot 20$ & \\
\hline $4 \mathrm{~s} \rightarrow 4 \mathrm{p}\left(2 \mathrm{~s}_{4} \rightarrow 3 \mathrm{p}_{3}\right)$ & $32 \cdot 6$ & & & & 35.4 & $34 \pm 17$ \\
\hline $4 \mathrm{~s} \rightarrow 4 \mathrm{p}^{\prime}\left(2 \mathrm{~s}_{4} \rightarrow 3 \mathrm{p}_{1}\right)$ & 2.08 & & & & 1.99 & $2 \cdot 6 \pm 1 \cdot 3$ \\
\hline $4 s^{\prime} \rightarrow 4 \mathrm{p}\left(2 \mathrm{~s}_{2} \rightarrow 3 \mathrm{p}_{3}\right)$ & $2 \cdot 75$ & & & & 1.99 & $2 \cdot 6 \pm 1 \cdot 3$ \\
\hline $4 s^{\prime} \rightarrow 4 p^{\prime}\left(2 s_{2} \rightarrow 3 p_{1}\right)$ & 31.9 & & & & 35.4 & $34 \pm 17$ \\
\hline
\end{tabular}

Table 7. Test for validity of the $J$-file sum rule (Shortley 1935).

\begin{tabular}{lccc}
\hline Array & A & B & C \\
\hline $3 \mathrm{~s} \rightarrow 3 \mathrm{p}$ & 6.75 & 7.0 & 6.75 \\
$3 \mathrm{~s} \rightarrow 4 \mathrm{p}$ & 0.11 & 0.18 & 0.04 \\
$4 \mathrm{~s} \rightarrow 3 \mathrm{p}$ & 7.17 & 11.31 & 6.06 \\
$4 \mathrm{~s} \rightarrow 4 \mathrm{p}$ & 35.4 & 34.0 & 37.4 \\
\hline
\end{tabular}

\footnotetext{
A, sum of the line strengths from table 6 for the transition $s_{4} \rightarrow p_{3}$ and $s_{2} \rightarrow p_{3}$. $\mathrm{B}$, sum of the line strengths from table 6 for the transitions $s_{4} \rightarrow p_{1}$ and $s_{2} \rightarrow p_{1}$. $\mathrm{C}, \frac{1}{7}$ th of the line strength from table 2 for the transition $s_{5} \rightarrow p_{9}$.
}

3.3.1. $3 s \rightarrow 3 p$ line strengths. These transitions provide the most rigorous test of our theoretical procedure since there are both the accurate experimental measurements of Bridges and Wiese (1970) and the accurate theoretical calculations of Koenig (1971) and Mehlhorn (1969) with which to compare our results. Except for the weakest transition $\left(3 s^{\prime} \rightarrow 3 p\right)$, our results are within the estimated error of the experimental measurements. Our overall agreement with experiment is comparable to that of Koenig (1971) who fits experimental energy levels using the effective operators of Trees (1952) and who also includes the effects of second-order perturbations. Our results are in somewhat better agreement with experiment than those of Mehlhorn (1969), who has done an intermediate coupling calculation by fitting $g$-factors as well as energy levels. Both of these calculations seem limited to low-lying excited levels (Aymar et al 1970) and must be performed for each configuration. (By contrast, the parameters we have obtained in tables 4 and 5 
by analysing entire Rydberg series allow us to calculate line strengths for any transition between the two Rydberg series involved.) We see from table 7 that the $J$-file sum rule holds fairly well for these transitions according to our calculations. This is also in agreement with the data of Bridges and Wiese (1970).

3.3.2. $3 s \rightarrow 4 p$ line strengths. We compare our results for the $3 s \rightarrow 4 p$ and $3 s^{\prime} \rightarrow 4 p^{\prime}$ transitions with the line strengths in Wiese et al (1966), which are based on the lifetime data of Klose (1966) for the $4 \mathrm{p}$ level. As with the $1 \mathrm{~s}_{5} \rightarrow 3 \mathrm{p}_{9}$ transition in table 2 , these experimental line strengths are not expected to be very accurate (Wiese et al 1966). Our line strengths for the $3 \mathrm{~s} \rightarrow 4 \mathrm{p}$ transition is a factor of two smaller than experiment. Our line strength for the $3 \mathrm{~s}^{\prime} \rightarrow 4 \mathrm{p}^{\prime}$ transition, however, is in agreement with experiment. We also present in table 6 line strengths for the two weak transitions $3 \mathrm{~s} \rightarrow 4 \mathrm{p}^{\prime}$ and $3 s^{\prime} \rightarrow 4 p$. There are no other data to compare with these. From table 7 we see that the $J$-file sum rule does not hold for these transitions according to our calculations.

3.3.3. $4 s \rightarrow 3 p$ line strengths. We compare our line strengths for these transitions with those obtained by Murphy (1968) by means of an intermediate coupling calculation. We see from table 6 that whereas the intermediate coupling calculation predicts equality of the two strong transitions and of the two weak transitions, our calculations do not. The total strength for the four transitions is predicted by our calculations to be much larger than that predicted by Murphy (1968). From table 7 we see that the $J$-file sum rule does not hold according to our calculations.

3.3.4. $4 s \rightarrow 4 p$ line strengths. We compare our line strengths for these transitions with the intermediate coupling calculations of Murphy (1968) and Ufford (1937), the latter being included in Wiese et al (1966). Our results are in good agreement with both of these calculations. We see also from table 7 that the $J$-file sum rule does hold to a good approximation according to our calculations.

\section{Conclusions}

We have calculated absolute line strengths for transitions in neon between levels belonging to two-channel Rydberg series using energy eigenfunctions determined by analysis of Lu-Fano plots. The theory of Lu and Fano has been extended in order to treat lowenergy levels. The parameters listed in tables 4 and 5 may be used to calculate line strengths other than those we have presented in table 6 by using the formulas of $\S 2$.

Except for the weak transition $1 s_{2} \rightarrow 2 p_{3}$, we have obtained excellent agreement with the accurate experimental line strengths of Bridges and Wiese (1970) for the $3 s \rightarrow 3 p$ transitions. Our calculations, which treat the variation of the radial matrix elements from one level to another of the same (initial and/or final) configuration, are in disagreement with the predictions of intermediate coupling calculations for the $3 \mathrm{~s} \rightarrow 4 \mathrm{p}$ and $4 s \rightarrow 3 p$ transitions, but are in good agreement with such calculations for $4 s \rightarrow 4 p$ transitions. We have also calculated line strengths for transitions between one channel Rydberg series and for the $3 \mathrm{~s} \rightarrow 4 \mathrm{p}$ transition obtaining a result that is one fifth the experimental line strength listed in Wiese et al (1969). Since the errors in these latter calculations of ours are almost entirely due to the Coulomb approximation, and since this error is estimated to be no more than a few per cent for the $3 \mathrm{~s} \rightarrow 4 \mathrm{p}$ transition, we suspect the accuracy of the experimental line strengths for these transitions. 


\section{Acknowledgments}

I wish to thank Dr K T Lu and Professor U Fano for clarifying numerous points in their work for me. I have also benefitted from conversations with Mr C M Lee. Lastly, I am most grateful to Professor Fano for his critical readings of the manuscript.

I was supported while at the University of Chicago by a National Science Foundation Graduate Fellowship and by the U.S. Atomic Energy Commission, Contract No C00-1674-47.

\section{Appendix: Renormalized effective quantum numbers}

As discussed in $\S 2.4$, the procedure for obtaining the parameters $\xi_{\alpha}$ and $U_{i \alpha}$ is complicated by the number of transformations needed between the set of $\mu_{\beta}, \mathfrak{U}_{i \beta}$ and $\xi_{\alpha}, U_{i \alpha}$. To avoid this complication we derive here a simple equation, involving $\xi_{\alpha}, U_{i \alpha}$, and renormalized effective quantum numbers, that may be fitted directly to experimental energy level data.

We start by expanding the sine in equation (2.8) and making the following substitutions: (i) we replace $\mathscr{A}_{\beta}$ by

$$
\mathscr{A}_{\beta}=\frac{1}{\sin \pi \mu_{\beta}} \sum_{i} \tilde{\mathfrak{U}}_{i \beta} B^{1 / 2}\left(v_{i}, l_{i}\right)\left(\sum_{\alpha} U_{i \alpha} \sin \pi \xi_{\alpha} A_{\alpha}\right)
$$

which is the inverse of equation (2.7a); (ii) we replace the left side of equation (2.7b) by the right side. The result is

$$
\sin \pi v_{i}\left(\sum_{\alpha} U_{i \alpha} \cos \pi \xi_{\alpha} A_{\alpha}\right)+\left(\cos \pi v_{i} B\left(v_{i}, l_{i}\right)+\sin \pi v_{i} G\left(v_{i}, l_{i}\right)\right)\left(\sum_{\alpha} U_{i \alpha} \sin \pi \xi_{\alpha} A_{\alpha}\right)=0 .
$$

We now multiply equation (A2) by an arbitrary function $F\left(v_{i}, l_{i}\right)$ and define renormalized effective quantum numbers $v_{i}^{\mathrm{R}}$ by the relations :

$$
\begin{aligned}
& F\left(v_{i}, l_{i}\right) \sin \pi v_{i} \equiv \sin \pi v_{i}^{\mathbf{R}} \\
& F\left(v_{i}, l_{i}\right)\left\{\cos \pi v_{i} B\left(v_{i}, l_{i}\right)+\sin \pi v_{i} G\left(v_{i}, l_{i}\right)\right\} \equiv \cos \pi v_{i}^{\mathbf{R}} .
\end{aligned}
$$

Note that unless all $l_{i}$ are the same, the newly defined quantities $v_{i}^{\mathrm{R}}$ will depend on $l_{i}$, which is not desirable since the ordinary $v_{i}$ do not depend on $l_{i}$. We determine the function $F\left(v_{i}, l_{i}\right)$ by requiring that $\cos ^{2}\left(\pi v_{i}^{\mathbf{R}}\right)+\sin ^{2}\left(\pi v_{i}^{\mathbf{R}}\right)=1$, that is

$$
F\left(v_{i}, l_{i}\right)=\left\{\sin ^{2}\left(\pi v_{i}\right)+\left(\cos \pi v_{i} B\left(v_{i}, l_{i}\right)+\sin \pi v_{i} G\left(v_{i}, l_{i}\right)\right)^{2}\right\}^{-1 / 2} .
$$

With this definition of $F\left(v_{i}, l_{i}\right)$, the result of multiplying equation $\mathrm{A} 2$ by $F\left(v_{i}, l_{i}\right)$ may be written as

$$
\sum_{\alpha} U_{i \alpha} \sin \pi\left(v_{i}^{\mathrm{R}}+\xi_{\alpha}\right) A_{\alpha}=0 \quad \text { for all } i .
$$

Equation (A5) is formally the same as equation (2.8) with the major difference that it is written in terms of the parameters $\xi_{\alpha}, U_{i \alpha}$. Proceeding as before, we may obtain equations analogous to equations (2.9-2.11). In particular, the equation corresponding to equation (2.11) is given by equation (2.14). This latter equation is the one we have fitted to experimental data in the numerical calculations presented in $\S 3$. 


\section{References}

Aymar M, Feneuille S and Klapisch M 1970 Nucl. Instrum. Meth. $90137-43$

Bates D R and Damgaard A 1949 Phil. Trans. R. Soc. A242 101-22

Bridges J M and Wiese W L 1970 Phys. Rev. A 2 285-93

Briggs J S 1971 Rev. mod. Phys. 43 189-230

Dehmer J L and Fano U 1970 Phys. Rev. A 2 304-9

Fano U 1970 Phys. Rev. A 2 353-65

Ham F S 1955 Solid State Physics Vol 1 eds F Seitz and D Turnbull (New York: Academic Press) pp 127-92

Herman F and Skillman S 1963 Atomic Structure Calculations (Englewood Cliffs, New Jersey: Prentice-Hall)

Hoffman D K, Raffenetti R C and Ruedenberg K 1972 J.math. Phys. 13 528-33

Kennedy J M and Cliff M J 1955 Transformation Coefficients Between LS and jj Coupling CRT-609 (Chalk River, Ontario: Atomic Energy of Canada Ltd)

Klose J Z 1966 Phys. Rev. 141 181-6

Koenig E 1971 Phys. Lett. 34A 284-6

Layzer D and Garstang R H 1968 Ann Rev. Astron. Astrophys. $6449-94$

Lu K T 1971 Phys. Rev. A 4 579-96

Lu K T and Fano U 1970 Phys. Rev. A $281-6$

Mehlhorn R 1969 J. Opt. Soc. Am. 59 1453-4

Moore C E 1949 Atomic Energy Levels Vol 1 (Washington DC: US Government Printing Office) 1970 Ionization Potentials and Ionization Limits Derived from the Analysis of Optical Spectra (Washington DC: US Government Printing Office)

Murphy P W 1968 J. Opt. Soc. Am 58 1200-8

Saraph H E and Seaton M J 1971 Phil. Trans. R. Soc. A271 1-39

Seaton M J 1958 Mon. Not. R. Astron. Soc. 118 504-18 1966 Proc. Phys. Soc. 88 801-14

Shortley G H 1935 Phys. Rev. 47 295-300

Trees R E 1952 Phys. Rev. 85382

Ufford C W 1937 Astrophys. J. 85 249-250

Weiss A W 1970 Nucl. Instrum. Meth. 90 121-31

Wiese W L, Smith M W and Glennon B M 1966 Atomic Transition Probabilities Vol 1 (Washington DC: US Government Printing Office) pp 126-9 